\title{
RESEARCH - IN ELEMENTARY PARTICLE PHYSICS
}

Technical Progress : Report

Lawrence E. Kirsch and Howard.J. Schnitzer

Department of Physics

Brandeis University

Waltham, Mass achusetts. 02154

May $31,1975-\div$ May 29,1976

PREPARED FOR THE U.S. ENERGY

RESEARCH AND DEVELOPMENT ADMINISTRATION

UNDER CONTRACT NO. E(11-1) 3230.
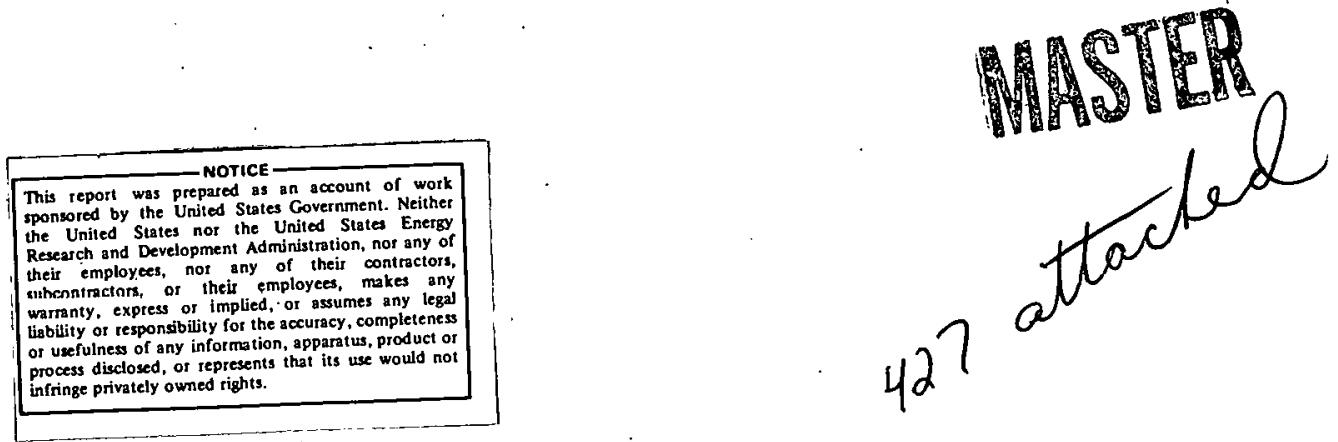


\section{DISCLAIMER}

This report was prepared as an account of work sponsored by an agency of the United States Government. Neither the United States Government nor any agency Thereof, nor any of their employees, makes any warranty, express or implied, or assumes any legal liability or responsibility for the accuracy, completeness, or usefulness of any information, apparatus, product, or process disclosed, or represents that its use would not infringe privately owned rights. Reference herein to any specific commercial product, process, or service by trade name, trademark, manufacturer, or otherwise does not necessarily constitute or imply its endorsement, recommendation, or favoring by the United States Government or any agency thereof. The views and opinions of authors expressed herein do not necessarily state or reflect those of the United States Government or any agency thereof. 


\section{DISCLAIMER}

Portions of this document may be illegible in electronic image products. Images are produced from the best available original document. 


\section{TECHNICAL PROGRESS REPORT \\ May 1,1975 to Apri1 30,1976}

Under this contract, research has been performed on both the theoretical and experimental properties of elementary particles. A brief description of work which has either been completed or is in progress is given below:

\section{EXPERIMENT}

I Multiparticle Spectrometer Progress

The Multiparticle Spectrometer (MPS) facility at Brookhaven has been the focus of the Brandeis experimental effort during the last year. This group has contributed to the general development of this unique device as well as participating in two of the first four experiments to be run. These experiments, which have completed their data acquisition are described in Sections $A$ and $B$ below. In addition we have made hardware and software advances described in Section $D$ which are necessary, not only for the present experiments, but for future work at the MPS.

\section{A. $\quad \bar{p} \rightarrow V^{\circ}+$ Neutrals}

We have completed 200 hours of data acquisition on an experiment to study the reaction $\bar{p} p \rightarrow V^{\circ}+$ Neutrals at $2.96 \mathrm{GeV} / \mathrm{c}$. Final states which include $\bar{\Lambda}^{\circ} \Lambda^{\circ}, \mathrm{K}^{\circ} \overline{\mathrm{K}}^{\circ}, \bar{\Lambda}^{\circ} \mathrm{K}^{\circ} \mathrm{n}$, will provide information on the dynamics of production through a measurement of polarizations and correlations.

Although the momentum of each beam particle is measured to $0.2 \%$ in a spectrometer, the beam has a momentum dispersion which spans a center of mass energy range of $\sim 100 \mathrm{MeV} / \mathrm{c}^{2}$. The central value corresponds to $2755 \mathrm{MeV} / \mathrm{c}^{2}$ which is the reported location of a possible 
pseudoscalar partner of the new narrow $J / \psi$ particle. Anomalies in the angular distribution of the $V^{\circ}$ 's would provide additional support for the existence of this state.

The data were taken at the MPS with: the liquid hydrogen target surrounded by a cylindrical proportional wire chamber (PWC) and a set of five:cylindrical spark chambers. Beyond these were three modules of capacitive spark chambers. A veto counter surrounding the target was used in coincidence with the appearance of $2-4$ tracks in the down-. stream PWC's as a trigger for the apparatus.

The raw flux corresponds to a sensitivity of approximately 2000 events/ $\mu \mathrm{b}$ and our on-line analysis indicates the production of at least $25,000 \cdot \bar{\Lambda}^{\circ}$ 's. The data will be processed during the coming months.

This experiment is a collaboration among Brandeis, University of Cinçinnati, and Syracuse University.

\section{B High Mass $K^{*}$ Production}

Data acquisition has been completed for a study of high mass $K^{*}$ production via the reaction $\mathrm{K}^{-} \mathrm{p} \rightarrow \pi^{+} \mathrm{K}^{-} \mathrm{n}$ at $6 \cdot \mathrm{GeV} / \mathrm{c}$ incident momentum. The MPS target region was filled with open framed planar capacitive chambers aligned parallel to the beam direction. The trigger system utilized.a water Cerenkov counter and: PWC's to select two particle final states for which a proton was absent... By demanding a large opening angle between the $\pi^{+}$and $\mathrm{K}^{-}$, we achieved an acceptance which increased.with high effective mass in contrast to conventional spectrometers.

Since this was the first MPS experiment utilizing spark chambers in the target.region, a preliminary run was used to evaluate software under operational conditions, to compare calculated and observed 
trigger rates, and to indicate possible hardware modifications.

The experimental data were obtained in a single subsequent run. The effective sensitivity of the data is approximately 100 events/ $\mu \mathrm{b}$. Preliminary processing has been completed on $25 \%$ of this data and shows clear evidence for $K^{*}(1420)$ production; as well as providing high sensitivity in the $K^{*}(1760)$ mass region, where the existence of a new state is the subject: of some uncertainty.

This experiment is a collaboration among Brandeis, Brookhaven, University of Massachusetts, and University of Pennsylvania.

C. Charm Particle Search

Glashow had proposed the existence of a pseudoscalar meson ${ }^{n_{c}}$ composed of $c \bar{c}$ quarks which 1 ay $30-80 \mathrm{MeV} / \mathrm{c}^{2}$ below the mass of the $\psi(3105)$. Thus we have taken data to try to observe the formation of this state in the reactions

$$
\begin{array}{r}
\mathrm{pp} \rightarrow \Lambda^{\circ}+\text { neutrals } \\
\rightarrow \mathrm{K}^{\circ}+\text { neutrals }
\end{array}
$$

in the mass range $2: 99-3.14 \cdot \mathrm{GeV} / \mathrm{c}^{2}$. A mass resolution of $\Delta M=3 \mathrm{MeV} / \mathrm{c}^{2}$ resulted in a sensitivity $\int_{\Delta M} \sigma(M) d M<40 \mathrm{MeV}-\mu \mathrm{b}$ (90\% confidence) for reaction (1) and $150 \mathrm{MeV}-\mu \mathrm{b}$ for reaction (2).

The mass region was spanned by three runs with central beam momenta of $3.83,3.95$, and $4.06 \mathrm{GeV} / \mathrm{c}$. The absolute value of each beam track was measured by a beam spectrometer to an accuracy of $0.2 \%$.

Since $\bar{\Lambda}^{\circ}$ production from reaction (1) is both peripheral and a slowly varying function of $s$, we can exhibit the results of the experiment as a search for structure in the ratios 


$$
\begin{aligned}
& \mathrm{R}_{1}=\text { No. of } \Lambda / \text { No. of } \bar{\Lambda} \\
& \mathrm{R}_{2}=\text { No. of } \mathrm{K}^{\circ} \text {. No. of } \bar{\Lambda}
\end{aligned}
$$

No significant effects are observed. Assuming isotropic decay for the pseudoscalar $n_{c}$ we obtain the limits given above.

This experiment was a collaboration among all the initial contributors, to the MPS including Brandeis, Brookhaven, City College of New York; Carnegie Mellon, University of Cincinnati, University of Massachusetts, University of Pennsylvania; Southeastern Massachusetts, and Syracuse University.

D. MPS Deveiopment 1. Capacitive Chambers --

Target Region I is a system of 38 spark chambers and three proportional wire chambers being developed jointly by. BNL and Brandeis. Currently 22 of these chambers in six modules are installed and: working in the MPS. These chambers were used by both of the experiments des-: cribed above. The full set of chambers will be mounted and: tested by the end of the summer. Of the sixteen remaining spark chambers about half have been individually tested and are ready for assembly into modules. The rest are in various stages of winding and fitting.

A delay of several months was experienced when we attempted to. use a copper clad aluminum wire. After winding several chambers some spools of the wire proved to be subject to corrosion and formation of metallic salts. Since this wire was too unstable for use in the MPS, all the affected chambers were cleaned and rewound with copper wire. 


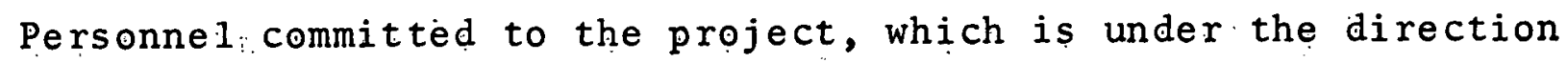
of. J. Bensinger, include one BNL physicist and two technicians, and one Brandeis technician .

\section{Capacitive Chamber Software:--}

We have developed a set of programs for unpacking and error testing the data from the capacitive spark chambers. It has been designed to run in several modes of operation: On-1ine during an. experiment with full display and monitoring features, off-line during processing, and in a test-mode for the testing of new capacitive modules. In addition, it is easily convertible to our PDP15 at Brandeis.

Another set of programs has been developed, for internal alignment of the capacitive spark chamber planes, as well as external alignment with; respect to the magnetostrictive read-out.planes using elaștịc scattering events.

\section{System Software --}

Software has been: developed for the alignment of the Beam PWC's as well as measurement of the beam momentum by a constrained fit. In addition the program is capable of assigning the correct spark to a beam track when multiple sparks occur in these chambers, thus increasing the number of useful events by as much as $20 \%$. There is also currently a feasability study for extending this procedure by tracing, beam: tracks through a quadrupole doublet to the two PWC!s in front of the MPS, in order, to solve a similar problem.

4. Pattern Recognition Software --

We have developed a new algorithm for pattern recognition in both the target region and downstream planar spark, chambers. Previously, the observation that particle trajectories were approximately a straight line in chambers which measure parallel to the magnetic field was the 
basis of the pattern recognition algorithm. Since no chambers of this kind exist in the target region, the algorithm has been changed to search for circles using triplets of sparks in chambers which measure perpendicular to the magnetic field. Due to the:field inhomogenities, the entire itrack is not a circle. Thus the radius found in the triplet most distant from the target is corrected by the approximate field change and then projected to the next:plane. The new:spark and the preceding pair now form a new triplet and the process continues. This approach is also successful in the downstream region when the tracks fail: to separate in the conventional view.: For the higher energy experiments to be: conducted in 1977, this technique should recover a large number of events.

These programs have been developed on, the Brandeis PDP-15. 5. Geometry Program --.

We have completed development of a program which does fit using the measured spark positions and errors, to determine the momenta and position of tracks. in the MPS。The program is completely general with respect to the type of chamber (planar or:cylindrical) and with respect to its orientation in the magnet..coordinate sys tem. This is required for analysis of: the data described above since a track may go through three different types of chambers, each with different measurement errors。. In addition the program uses the detailed map of the magnetic field to calculate the deviation of each spark from the particle trajectory and can reject sparks found by pattern recognition which do not belong to the track or add missed sparks. The program can also link track segments found by pattern recognition in different regions of the MPS or in different types of detectors. 
The basic algorithm is: a five parameter linear. fit to a helix in space using a chi-square minimization procedure which takes account of the non-uniform magnetic field.

Vertex finding routines then construct production and/or decay vertices using a general helix-helix intersection method.

II. Search for $\equiv *$ Production in $\mathrm{K}^{-}$P Interactions at $2.87 \mathrm{GeV} / \mathrm{c}$

Over: the past several years we have worked on a search for $\equiv$ : resonances in two large bubble chamber exposures: $K^{-}$in hydrogen with a sensitivity of $30 \mathrm{ev} / \mu \mathrm{b}$ and $\mathrm{K}^{-}$in deuterium with a sensitivity of 18 ev/pb. A summary paper on $K^{-} p$ interactions, combining data from both experiments, is nearing completion: We find evidence for four resonances:

1. $A \equiv \equiv^{*}(1820)$ with decay modes $\Lambda K^{-}, \equiv *(1530) \pi$ and $\equiv^{-} \pi^{+}$. The latter is not as firmly established because the $\equiv^{-} \pi^{+}$mass spectrum can be fit by are broad resonance instead of $\equiv *(1820)+$ $\equiv *(1960)$ Previously we had also observed the $\equiv *(1820)$ in $\mathrm{K}^{-} \mathrm{n}+\Lambda \mathrm{K}^{-} \mathrm{K}_{1}^{\circ}$

2.: $\mathrm{A} \equiv *(1860)$ with decay modes $\Lambda \bar{K}^{\circ}$ and $\Sigma \bar{K}^{\circ} \overline{\mathrm{K}}^{\circ}$. Although no signal is seen in $\Sigma^{\circ} K^{-}$, the upper 1 imit is on $1 y$ one standard deviation below that predicted from the $\Sigma^{-} \overline{\mathrm{K}}^{\circ} \cdot \operatorname{mode}$.

3. A: $\Xi^{*}(1960)$ with decay modes $\equiv^{-} \pi^{+}$and $\equiv *(1530) \pi$. We also observe a high-mass excess of events in the $\equiv^{\circ} \pi^{-}$combined-mass of the $\equiv^{\circ} \pi^{-} \mathrm{K}^{+}$. final.state that could be attributed to $\equiv *(1960)$ decay.

4. The controversial $\equiv *(1630)$ remains with a statistical signifịcance of three standard deviations Although the $\mathrm{K}^{-} \mathrm{p} \rightarrow \equiv^{-} \pi^{+} \mathrm{K}^{\circ}$ reaction in the deuterium separately yields an excess of events in the $\equiv^{*}(1630)$ region, this amounts to only two standard deviations. 
We thus find evidence for three separate resonances with at least two decay modes each in the $\equiv *$ mass region between 1.8 and $2.0 \mathrm{GeV} / \mathrm{c}^{2}$; where resonances with a wide variety of masses and widths have been claimed.

We have also measured upper limits for resonance production in those final states where no obvious signal is present. With the addition of more data, the $\equiv *(1630)$ persists but has not improved.

Cross-sections greater than $1 \mu b$ for production of $\equiv K(\pi)$ and YKK( $\pi$ ) final states have also been: measured.

We conclude that in order to obtain more detailed information on the $\equiv *$ resonant states requires an experiment at this energy with an order of magnitude of greater sensitivity

This work is a collaboration among Brandeis, Maryland, Syracuse and Tufts Universities.

\section{Technical Development}

With a long term commitment to the MPS program, it has become clear that the bulk of our computing activities will be transferred to the CDC7600 facility at.Brookhaven. No other computer can reasonably handle the: volume of data generated by the MPS. Therefore, a high speed data link to this system was required. The PBP-15 in our laboratory is equipped with a 2000 baud. synchronous transmission capability, however no software existed to match the INTERCOM communication protocol used at Brookhaven.

We have developed a system. which can use this hardware to act as a remote terminal to any Control Data System using this protocol. Since these systems are used in several national laboratories, this may be of general interest. Moreover the code is about $80 \%$ machine independent, so that it could be converted for use on other computers. 


\section{THEORY}

The theoretical physics program supported by the present contract can be conveniently divided into two principal research topics: 1) the analysis of meson spectroscopy, with special emphasis on the charmonium system, by means of a simple phenomenological.model, incorporating the ideas of quark confinement as abstracted from gauge theories; and 2) the further development of methods in quantum field: theory which avoid,a conventional perturbation expansion in, the coupling constant: The first: set of topics was motivated by the striking discovery of new particles by the SLAC and MIT groups. Our research, involving non-perturbative methods in field theory is part of an extensive ongoing program which began in 1973 with the discovery that the Yang-Mills gauge:mesons Reggeize in renormalizable gauge theories.: The techniques which have subisequently evolved, such as the $1 / \mathrm{N}$ expansion; have been particularly fruitful, with significant: advances being made during the last year. New results were obtained in other areas of field theory as well, although the topics do not fit neatly into these two main areas. A summary of this research follows.

\section{Charmonium Spectroscopy.}

The favored interpretation of the $\psi(3100)$ and $\psi^{\prime}(3700)$ mesons discovered in $\mathrm{e}^{+} \mathrm{e}^{-}$annihilation experiments is that they are the $n=1,{ }^{3} S_{1}$ and $n=2,{ }^{3} S_{1}$ bound-states of quark-antiquark pairs; with; the quark having "hidden charm". This system, called charmonium, is expected.to. have a number of spectroscopic features in common with that of positronium, although quark confinement and:strong forces, with their underlying gauge structure, lead to important differences . 
During the previous year, Kang and Schnitzer and others, developed a phenomenological analysis of charmonium spectroscopy based on a nonrelativistic potential model with a linear potential confining the quarks.: Since the identification of $\psi^{\prime}(3700)$ and: $\psi^{\prime \prime}(4200)$ as the first and second radial excitations of $\psi(3100)$ had been challenged by several workers, Kang and Schnitzer reexamined the original, assignments within the context of the linear potential models. It was their: conclusion that this assignment of $\psi^{\prime}$ and $\psi^{\prime \prime}$ must be retained if the interaction energy per unit length is universal for quark-antiquark pairs, i.e. if the linear potential is $S U(n)$ invariant in a quark model with $n$ flavors. If the potential differs for each meson system, then one may abandon the original assignment of $\psi^{\prime}$ and $\psi^{\prime \prime}$, but at the expense of making the charmonium spectrum relativistic.

Investigation of the leading relativistic corrections to the basic non-relativistic model was carried out, guided by phenomenological considerations. It was assumed that the leading spin-dependent corrections to the charmonium spectrum could be obtained from the Breit-Fermi Hamiltonian, but with the radial dependences derived from derivatives of the spin-independent phenomenological potential which confines the quarks. Predictions were obtained for the $\psi-n_{c}$ mass splitting of at most $100 \mathrm{Mev}$, and of the level splitting of the charmonium $\mathrm{P}$ states in the 3400-3550 Mev region. The first is in disagreement with the mass of the state tentatively identified as $n_{C}$, at $2800 \mathrm{Mev}$, while the definitive identification of the quantum numbers of the $P$ states in the 3400-3550 region awaits further experimental work. 
II - Non-Perturbative Methods

An extensive analysis of the $1 / \mathrm{N}$ expansion of $O(N)$ symmetric $\lambda \phi^{4}$. theory in four dimensions showed it to be a consiștent approximation method. It was shown that the ground state of the theory is $O(N)$ symmetric, and that spontaneous symmetry breaking is not possible in the large N limit. It was shown that the Green's functions are free of tachyons if constructed relative to. this ground state: The formalism of the $1 / \mathrm{N}$ expansion was extended to arbitrary $O(N)$ symmetric scalar field theories with non-derivative couplings, to leading order in $N$, and for any space-time dimension. The generality of the approach permitted the examination of a number of issues relevant to non-renormalizable theories.

Kang examined a gauge theory with $U(N)$ symmetry in the large $N$ limit, to all orders in the scalar field self-coupling, and to. lowest non-trivial order in the gauge coupling. There is a region in the coupling-constant space for which symmetry is broken spontaneously involving two separate phase transitions; one of the first kind and one of the second kind. This phenomenon persists for arbitrarily smali. (finite) gauge coupling. The vector-scalar boson mass ratio $\mathrm{m}_{\mathrm{v}}^{2} / \mathrm{m}_{\mathrm{s}}^{2}$. is of $O\left(\mathrm{~g}^{2}\right)$, which is in contrast to the Higgs or Coleman-Weinberg symmetry breaking mechanisms for which $\mathrm{m}_{\mathrm{v}}^{2} / \mathrm{m}_{\mathrm{s}}^{2}$ is $O(1)$ and $O\left(\mathrm{~g}^{-2}\right)$ respectively, where:g is the gauge coupling constant.

Townsend has carried out an extensive study $\phi^{6}$ theory in threedimensions, which is a renormalizable theory. A technique was developed which:allows the derivation of all the Green!s functions, and the effective potential to leading order in $1 / \mathrm{N}$. The global ground state of the model was found, and symmetry breaking was studied, which is pos sible for certain values of the free parameters. The effective 
potential was also evaluated in next-to-leading order in $1 / N$. It was found that no tachyons appear in the theory in the first two orders of the $1 / \mathrm{N}$ expansion. The conclusion is that the $1 / \mathrm{N}$ expansion appears to be, a consistent approximation scheme in both three and four dimensions :

Work has begun on combining results obtained from the large $\mathrm{N}$ ex: pansion with the semi-classical bound-state formalism of Dashen, Hasslacher, and Neveu (DHN). Abbott has considered the bound states of the two-dimensional $O(N)$ model, while work, in progress is concerned with the extension of the DHN techniques to four dimensions. Present efforts are: concentrated on developing the trace identities and mode sums required for this extension of the DHN method.

\section{Other Results}

Kang has studied the uniqueness of renormalized quantities in dimensionaly regularized field theory. It was proved that in any order of the perturbation expansion, the dimensionally regularized Feynman integrations may be multiplied by an arbitrary analytic function of the space-time dimension which can be absorbed in the redefinition of the bare coupling constants so that the finite renormalized quantities are unchanged.

Following a suggestion of: Weinberg, Abbott studied the massless representations of the Poincaré group which include states with:continuous spin indices. Free field:operators corresponding to these states were constructed, and were shown to obey non-causal commutation or anticommutation relations. 
PUBLICATIONS FRÓM APRIL 1, 1975 TO APRIL 30, 1976

\section{Experimenta1}

1. "Search : for $\equiv *$. Resonances in $2.87 \mathrm{GeV} / \mathrm{C} \mathrm{K} \cdot \mathrm{n}$. In teractions!" (with Univ. of Maryland, Syracuse, and Tufts University), Phys. Rev。 D 12 , 1859 (1975).

2. "Cascade, Final States in $K^{-n}$. Interactions at $2.87-\mathrm{GeV} / \mathrm{c}, "$ Ellen Briefel, PhD Thesis.

3. "Reaction $\pi^{+} d \rightarrow \pi^{+} \pi^{+}{ }^{-} d$ at $15 \mathrm{GeV} / \mathrm{C}^{\prime \prime}$ (with:Florida State), Phys Rev. D11, 996 (1975).

4. "Search for Charmed Mesons and Baryons". (with Florida State), Phys: Rev. Letters $\underline{36}, 296 \cdot(1975)$ :

\section{Theoretical}

H.J. Schnitzer

5. "Dynamics of Light and Heavy Bound Quarks"' (with J.S. Kang), Phys。 Rev.D走, 841 (1975):

6. "Is $\psi^{\prime}(3700)$ a Radical Excitation of $\psi$ (3100)?" (with J.S. Kang), Phys. Rev. D12, 2791 (1975).

7. "Hyperfine Splitting of Ground-State Charmonium," Phys. Rev. B13, 74 (1976).

8. "P States of Charmonium and the Forces that Confine Quarks," Phys. Rev。 Letters $\underline{35}, 1540$ (1975).

9. "Bound States, Tachyons, and the Restoration of Symmetry in the $1 / \mathrm{N}$ Expansion" (with L.F. Abbott and J.S. Kang), Phys。Rev. D $\underline{13}$, April 15, 1976. 
10. "The 1/N Expansion of Renormalizable and Non-Renormalizable Scalar Field: Theories," Nuclear Physics:B (to be published). J.S. Kang:

11. "Uniqueness of Renormalized Quantities in Dimenșional Regularization,": Phys.: Rev. D13, 851 (1976).

12: "Spontaneous Symmetry Breakdown, in a $U(N)$ Gauge Model in the $1 / N$ Expansion," Brandeis : University preprint, Apri1 1976.

P.K: Townsend

13. "Spontaneous Symmetry Breaking in $O(N)$ Symmetric. $\phi^{6}$ Theory in. the 1/N Expansion," Phys. Rev. D12, 2269 (1975).

14. "Consistency of the 1/N Expansion for Three Dimensional: $\phi^{6}$ Theory,". submitted to Nuclear Physics $B$.

15. "The Global Ground State of $\phi^{6}$-Theory in Three, Dimensions," sub-: mitted to Phys: Rev. D.

16. "The 1/N Expansion of Scalar Field Theories," PhD Thesis, April 1976 .

L:F:Abbott

17. "Massless Particles with Continuous Spin: Indices," Phys . Rev. D13, Aprit 15, 1976 .

18. "Bound States of the Two-Dimensiona1: $O(N)$ Mode1," submitted to Phys. Rev. D. 